\title{
LAWS OF INFORMATION IN DECENTRALIZED CONTROL SYSTEMS
}

\author{
Luboš POPOVIČ, Ján SARNOVSKÝ, Vratislav HLADKÝ \\ Department of Cybernetics and Artificial Intelligence, Faculty of Electrical Engineering and Informatics, \\ Technical University of Košice, Letná 9, 04200 Košice, Slovak Republic, tel.: +421 55602 4214, \\ e-mail: lubos.popovic@tuke.sk, jan.sarnovsky@tuke.sk,vratislav.hladky@tuke.sk
}

\begin{abstract}
This paper deals with the laws of information in decentralized control systems. In the case of decentralized control of complex systems, often referred to hierarchical systems, theoretically analyze the possibility of using an n-dimensional information theory to deal with such systems. N-dimensional information theory is an interesting tool to deal with these systems. On the basis of information theory the partition laws of information rates are listed. These laws are fundamental requirements for complex systems that must be met and by their quantification one can be able to compare these complex control systems and track how different parts of the system communicate and collaborate with others, etc. In the final section the deterministic partition law of information rates and its possibility of use for dynamical systems are presented.
\end{abstract}

Keywords: cybernetics, entropy, information, variety, probability, regulator, decentralized control

\section{INTRODUCTION}

Control systems are becoming increasingly interesting from the perspective of information structure and information flow between various parts of systems. American scientist - the pioneer of cybernetics W. Ross Ashby in his book Introduction to cybernetics presented a different view on controllers and controlled systems as is now generally used. Ashby's approach is based on the theory of information, where systems are regarded as transmission channels, which are capable to transfer different amounts of information. His work is among the experts of this field, highly prized today. Based on similar considerations and simple examples he worked on in the world well known law of requisite variety [1]. Its importance for the theory of automatic control is very significant. It is therefore necessary to devote sufficient attention. For its verification it is necessary to deal with information theory, probability and entropy, since variety is very closely related to entropy. W. Ross Ashby formulated this law for centralized control systems, but it is interesting that a similar principle can be also applied to systems with hierarchical structure, for example to decentralized control systems. In this regard continued his student Roger C. Conant, and he formulated laws of information's, which control such systems [2]. His major contribution was the decomposition of systems into subsystems by using theory of information. The only one disadvantage is that these laws are not quite good applicably in the study and analysis of dynamic systems. The role of this paper is to remove this disadvantage and modify these laws for use in decentralized dynamical systems and verify its on a selected example of a complex system.

\section{PRINCIPLE OF REQUISITE VARIETY}

The subject of regulation is very wide in its applications, covering as it does most of the activities in physiology, sociology, ecology, economics, and much of the activities in almost every branch of science and life. Further, the types of regulator that exist are almost bewildering in their variety. Let us therefore forget all about regulation and simply suppose that we are watching two players, $R$ and $D$, who are engaged in a game (see Table 1):

Table 1 Table of game for two players

\begin{tabular}{|c|c|c|c|c|}
\hline & \multicolumn{3}{|c|}{$R$} \\
\hline & & $\alpha$ & $\beta$ & $\gamma$ \\
\hline \multirow{3}{*}{$D$} & 1 & $b$ & $a$ & $c$ \\
\hline & 2 & $a$ & $c$ & $b$ \\
\hline & 3 & $c$ & $b$ & $a$ \\
\hline
\end{tabular}

$D$ must play first, by selecting a number, and thus a particular row. $R$, knowing this number, then selects a Greek letter, and thus a particular column. The italic letter specified by the intersection of the row and column is the outcome. If it is an $a, R$ wins; if not, $R$ loses. Examination of the table soon shows that with this particular table $R$ can win always. Whatever value $D$ selects first, $R$ can always select a Greek letter that will give the desired outcome. $R$ has, complete control of the outcome [3].

From all possible tables let us eliminate those that make $R$ 's game too easy to be of interest. Let us consider, then, only those tables in which no column contains a repeated outcome. When this is so $R$ must select his move on full knowledge of $D$ 's move; i.e. any change of $D$ 's move must require a change on $R$ 's part (see Table 2) [3].

In the case if outcome is letter $k$, then we have transformation which uniquely specifies a set of outcomes. It can now be stated that the variety in this set of outcomes cannot be less than $D$ 's variety/R's variety i.e., in this case $9 / 3$. In general if no two elements in the same column are equal, and if a set of outcomes is selected by $R$, one from each row, and if the table has $r$ rows and c columns, then the variety in the selected set of outcomes cannot be fewer than $\mathrm{r} / \mathrm{c}$.

We can now look at this game still with the restriction that no element may be repeated in a column from a slightly different point of view. If the varieties are measured logarithmically, and if the same conditions hold, 
Table 2 Table of game for two players, where no column contains the same two results

\begin{tabular}{|c||c||c|c|c||}
\hline \multicolumn{2}{|c|}{} & \multicolumn{3}{c||}{$R$} \\
\cline { 2 - 4 } \multicolumn{1}{|c|}{} & $\alpha$ & $\beta$ & $\gamma$ \\
\hline \hline \multirow{5}{*}{$D$} & 1 & $f$ & $f$ & $k$ \\
\cline { 2 - 5 } & 2 & $k$ & $e$ & $f$ \\
\cline { 2 - 5 } & 3 & $m$ & $k$ & $a$ \\
\cline { 2 - 5 } & 4 & $b$ & $b$ & $b$ \\
\cline { 2 - 5 } & 5 & $c$ & $q$ & $c$ \\
\cline { 2 - 5 } & 6 & $h$ & $h$ & $m$ \\
\cline { 2 - 4 } & 7 & $j$ & $d$ & $d$ \\
\hline & 8 & $a$ & $p$ & $j$ \\
\hline & 9 & $l$ & $n$ & $h$ \\
\hline \hline
\end{tabular}

then the theorem takes a very simple form. Let $V_{D}$ be the variety of $D, V_{R}$ that of $R$, and $V_{O}$ that of the outcome (all measured logarithmically). Then the previous section has proved that $V_{O}$ cannot be less, numerically, than the value of $V_{D}-V_{R}$. Thus $V_{O}$ 's minimum is $V_{D}-V_{R}$. If $V_{D}$ is given and fixed, $V_{D}-V_{R}$ can be lessened only by a corresponding increase in $V_{R}$. Thus the variety in the outcomes, if minimal, can be decreased further only by a corresponding increase in that of $R$. This is the law of Requisite Variety: only variety in $R$ can force down the variety due to $D[4]$. Only variety can destroy variety.

\section{PROBABILITY, ENTROPY AND MUTUAL INFORMATION}

Probability, entropy and mutual information or transmissions are the main part of information theory. Information theory is science investigating quantitative laws related to transfer and processing of information. At the present, the theory of information is necessary mathematical tool for the study of various control processes, what is often forgotten. Receiving, processing, transmission and storage of information of various kind is the main task of each control system. To solve different tasks of information theory it is necessary to learn how quantitative measure the content of information which is transmitted or received through the communication channel.

\subsection{Probability}

Let's look at the system, which is able to receive final amount of states $x_{1}, x_{2}, \ldots, x_{n}$ with probabilities $p_{1}, p_{2}, \ldots, p_{n}$, where

$p_{i}=P\left(X \sim x_{i}\right)$,

is probability, that system $X$ receive state $x_{i}$. It is clear that

$\sum_{i=1}^{n} p_{i}=1$

These data can be written in table form (see Table 3), where in the first row are states of the system and in the second row are corresponding probabilities [5].
Table 3 Probability table

\begin{tabular}{||l||l|l|l|l||}
\hline \hline$x_{i}$ & $x_{1}$ & $x_{2}$ & $\cdots$ & $x_{n}$ \\
\hline$p_{i}$ & $p_{1}$ & $p_{2}$ & $\cdots$ & $p_{n}$ \\
\hline
\end{tabular}

This table is identical with table table of random discrete variable with values $x_{1}, x_{2}, \ldots, x_{n}$ and their probabilities $p_{1}, p_{2}, \ldots, p_{n}$.

\subsection{Entropy}

As a measure of uncertainty of the system (or discrete variable) $X$ in information theory is special characteristic called entropy. Entropy is main concept of information theory. Entropy of the system is sum of products of probabilities of different states and the logarithms of these probabilities with the opposite sign

$H(X)=-\sum_{i=1}^{n} p_{i} \log _{a} p_{i}$

The main properties of entropy are: entropy is equal to zero, if one of the states is certain and others are impossible; entropy has maximum if all states of the system are equally probable. Consider the simplest system $X$, which has two equally probable states (see Table 4).

Table 4 Probability table of system with two equally probable states

\begin{tabular}{|l||c|c|}
\hline$x_{i}$ & $x_{1}$ & $x_{2}$ \\
\hline$p_{i}$ & $\frac{1}{2}$ & $\frac{1}{2}$ \\
\hline
\end{tabular}

Actually entropy calculated by using (3) is $H(X)=-\left(\frac{1}{2} \log _{2} \frac{1}{2}+\frac{1}{2} \log _{2} \frac{1}{2}\right)=1$. Base unit for entropy is bit (from English word binary digit) [5].

\subsection{Mutual information}

Mutual information, transmission or transinformation is measure of amount by which two variables are related, i.e., are not statistically independent. Transmission between two variables is denoted by $T\left(X_{1}: X_{2}\right)$ and defined through probabilities or by

$$
\begin{aligned}
T\left(X_{1}: X_{2}\right) & =H\left(X_{1}\right)+H\left(X_{2}\right)-H\left(X_{1}, X_{2}\right) \\
& =H\left(X_{1}\right)-H\left(X_{1} \mid X_{2}\right) \\
& =H\left(X_{2}\right)-H\left(X_{2} \mid X_{1}\right)
\end{aligned}
$$

It is symmetric and measures the amount by which knowledge of one variable reduces uncertainty about other, or the amount by which the joint uncertainty $H\left(X_{1}, X_{2}\right)$ is smaller than it would be with $X_{1}$ and $X_{2}$ 
independent (then, $\left.H\left(X_{1}, X_{2}\right)=H\left(X_{1}\right)+H\left(X_{2}\right)\right)$. The transmission is therefore a measure of relatedness between variables, which accounts for its usefulness in systems science. $T\left(X_{1}: X_{2}\right)$ falls in the interval $\left[0, \min \left\{H\left(X_{1}\right), H\left(X_{2}\right)\right\}\right]$, being 0 if and only if $X_{1}$ and $X_{2}$ are statistically independent and maximum if and only if one variable determines the other [2].

\subsection{Method and examples for calculation of entropy}

For application of the principle of requisite variety for each control systems, it is necessary to design method of measurement and calculating of entropy. Like entropy of experiments, entropy of signals is also related with probability.

Let consider urn with 20 balls. 8 balls are white, 5 balls are black and 7 balls are red. Balls are well mixed. Balls are drawing out in random way and then put back into the urn. After several attempts, we found that each colour ball is drawing out with likelihood ratio as the ratio of the frequency representation of each colour. So the probability that white ball is drawing out is $8 / 20=40 \%$, the black ball is $5 / 20=25 \%$ and red ball is $7 / 20=35 \%$ (see Fig. 1).

Entropy of this experiment calculated by using (3) is $H=-0,4 \cdot \log _{2} 0,4-0,25 \cdot \log _{2} 0,25-0,35 \cdot \log _{2} 0,35=1,5589$ bit.

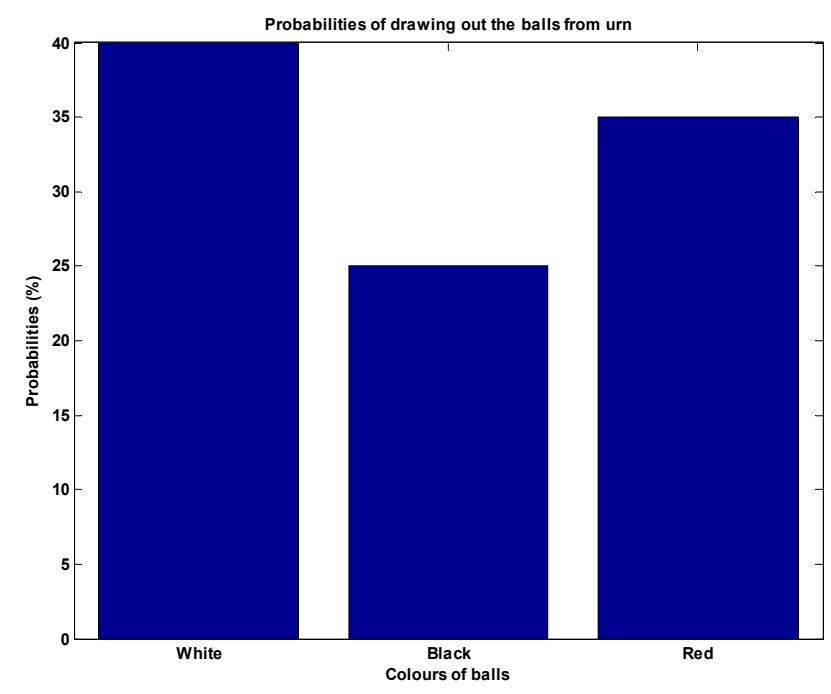

Fig. 1 Probability of drawing out the balls from urn

Consider now discrete signal with values 1,2 and 3 during 10 seconds with sampling time 1 second, according to this sequence $S=\{1,3,3,2,1,3,3,1,2,3\}$ (see Fig. 2).

Value 1 occurs in signal 3 times, value 2 occurs 2 times and value 3 occurs 5 times in signal. This occurs are in literature often called as multiplicity. From multiplicity is possible to calculate probabilities of values 1,2 and 3 . Probability for value 1 is $3 / 10$, for value 2 is $2 / 10$ and for value 3 is $5 / 10$. Entropy for this discrete signal with three values is $H=-\frac{3}{10} \cdot \log _{2} \frac{3}{10}-\frac{2}{10} \cdot \log _{2} \frac{2}{10}-\frac{5}{10} \cdot \log _{2} \frac{5}{10}=$
$=1,4855$ bit. In the left part of figure (see Fig. 2) is discrete signal and in the right part is histogram. Based on this histogram we can determine the frequency of specific value, then probability of specific value and so entropy of current signal.
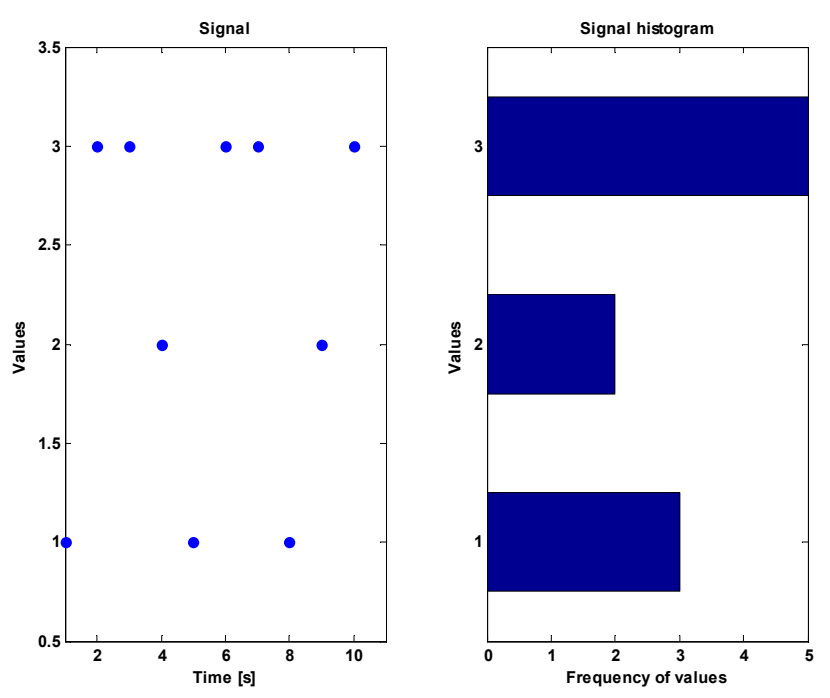

Fig. 2 Discrete signal with histogram

\section{DECENTRALIZED CONTROL SYSTEMS}

As complex systems we can consider systems that consist of large number of parts that are interacting with each other. Since certain difficulties arise in control such system as a whole, i.e. centralized, it is appropriate to use decomposition - particulate whole system to $N$ independent subsystems and control whole system as decentralized.

\subsection{Decomposition of complex system into subsystems}

Look now at complex system in terms of information theory. Since in most cases we deal with dynamical systems - systems that change their properties depending on time and information theory has no natural relationship to real time, it is necessary to add some additional considerations. The information theory can be used only in the event that we have measured real data from complex system.

After each sustained observation of a complex dynamic system, we get some protocol, which include values of observed variables and corresponding time

Table 5 Protocol of complex system

\begin{tabular}{||c||c||}
\hline \hline Time & States of system \\
\hline 0 & $\left(x_{1}^{0}, \ldots, x_{n}^{0}\right)$ \\
\hline 1 & $\left(x_{1}^{1}, \ldots, x_{n}^{1}\right)$ \\
\hline$\ldots$ & $\ldots$ \\
\hline$j$ & $\left(x_{1}^{j}, \ldots, x_{n}^{j}\right)$ \\
\hline$\ldots$ & $\ldots$ \\
\hline
\end{tabular}


vector. Thus, if the variables are $x_{1}, x_{2}, \ldots, x_{n}$, and the system, as $n$-tuple, was observed at times indicated by superscripts, the protocol will consist of an actual value given to each symbol (see Table. 5).

A most important case occurs when the system is state-determined, i.e. when $n$-tuple $x^{j+1}$ is the same function of $x^{j}$ whatever the value of $j$. The protocol can be represented equivalently by the single function $x^{j+1}=f\left(x^{j}\right)$, or $x^{\prime}=f(x)$ [6]. When this is so, an important new set of variables, $2 n$ in number, $\left(x_{1}, x_{2}, \ldots, x_{n}, x_{1}^{\prime}, x_{2}^{\prime}, \ldots, x_{n}^{\prime}\right)$ represents the transitions, i.e. the behaviour in real time, one state of the new system (of $2 n$ variables) corresponding to one transmission of the old.

Between these new $2 n$ variables all the various measures of entropy, transmission and interaction may be computed exactly as over any other set of variables, but they can now be interpreted by their relations with real time. Thus $T\left(x_{i}: x_{i}^{\prime}\right)$ measures something very close to concept of "cause and effect", for it measures how much the latter value at $x_{i}$ is dependent on the prior value. If larger sets are studied $\left(x_{1}^{0}, \ldots, x_{n}^{0}, x_{1}^{1}, \ldots, x_{n}^{1}, x_{1}^{j}, \ldots, x_{n}^{j}\right)$, such a transmission as $T\left(x_{i}^{0}: x_{i}^{j}\right)$ would measure how much the variable $x_{i}$ shows $j$ steps later in time, the effect of its earlier value. The measure thus catches something essential in the concept of $x_{i}$ 's "memory" [6].

\subsubsection{Example of the decomposition of complex system}

Consider now complex system according to [7], which can be write in the form

$$
\begin{aligned}
& \dot{x}_{1}(t)=x_{2}(t) \\
& \dot{x}_{2}(t)=-x_{1}(t)-x_{3}(t)+u_{1}(t) \\
& \dot{x}_{3}(t)=x_{4}(t) \\
& \dot{x}_{4}(t)=-x_{3}(t)-x_{1}(t)+u_{2}(t)
\end{aligned}
$$

Respectively in discrete form using forward difference (Euler method), we have $\dot{x}(t)=\frac{x(t+T)-x(t)}{T}$, then

$$
\begin{aligned}
& x_{1}(k+1)=x_{1}(k)+T x_{2}(k) \\
& x_{2}(k+1)=x_{2}(k)-T x_{1}(k)-T x_{3}(k)+T u_{1}(k) \\
& x_{3}(k+1)=x_{3}(k)+T x_{4}(k) \\
& x_{4}(k+1)=x_{4}(k)-T x_{3}(k)-T x_{1}(k)+T u_{2}(k)
\end{aligned}
$$

where $T$ is sampling interval equal to 0.1 seconds. After simple simulation of this system with initial conditions $\left[\begin{array}{llll}0.5 & 0.3 & -0.5 & -0.1\end{array}\right]$ and inputs represented with Heaviside functions, we get the protocol mentioned above with corresponding sample data, which represent the four state variables measured at corresponding sampling interval. On the following figure (see Fig. 3) is time behaviour of state variables of complex system for selected time period.
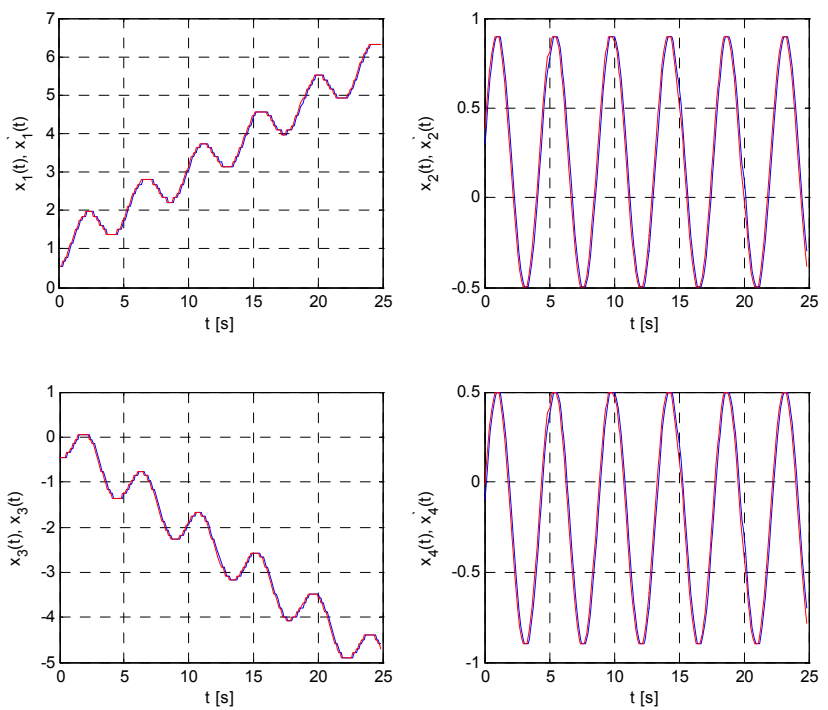

Fig. 3 Time behaviour of state variables with selected initial conditions and inputs

From these measured data we get sets of variables, generally labelled as $S_{j}$, where $S_{j}=\left\{x_{j 1}, x_{j 2}, \ldots, x_{j n_{j}}\right\}$. Then we can denote $H\left(S_{j}\right)$ as entropy of $S_{j}=\left\{x_{j 1}, x_{j 2}, \ldots, x_{j n_{j}}\right\}$. It is a nonnegative measure of the total amount of activity or variability in the set $S_{j}$, and it is calculated by a formula similar to (3). The entropy of the union of two sets $S_{i}$ and $S_{j}$ is denoted by $H\left(S_{i}, S_{j}\right)$. The observed transmission between $S_{i}$ and $S_{j}$ is denoted $T\left(S_{i}: S_{j}\right)$ and is defined as follows [8]

$T\left(S_{i}: S_{j}\right)=H\left(S_{i}\right)+H\left(S_{j}\right)-H\left(S_{i}, S_{j}\right)$

This is nonnegative measure of strength of the relation between $S_{i}$ and $S_{j}[8]$.

Suppose now a system is in fact " nearly decomposable" into subsystems $S_{1}, S_{2}, \ldots, S_{m}$, then it would be expect that the constraint holding between the subsystem over a short time span would be weak compared to the constraint within them. The constraint holding within the $j$-th subsystem $S_{j}=\left\{x_{j 1}, x_{j 2}, \ldots, x_{j n_{j}}\right\}$ will be measured reasonably by $T_{w j}$ defined as

$T_{w j}=T\left(x_{j 1}: x_{j 1}^{\prime}: x_{j 2}: x_{j 2}^{\prime}: \cdots: x_{j n_{j}}: x_{j n_{j}}^{\prime}\right)$

since this transmission measures nonindependance of all variables in the subsystem over time increment. The strength of the relation between the $i$-th and $j$-th subsystems over one time increment is measured by $T_{b i j}$, defined as

$T_{b i j}=T\left(\left\langle S_{i}, S_{i}^{\prime}\right\rangle:\left\langle S_{j}, S_{j}^{\prime}\right\rangle\right)$

and the constraint between all subsystem over one time increment is measured by $T_{b}$ 


$$
T_{b}=\left(\left\langle S_{1}, S_{1}^{\prime}\right\rangle:\left\langle S_{2}, S_{2}^{\prime}\right\rangle: \cdots:\left\langle S_{m}, S_{m}^{\prime}\right\rangle\right)
$$

In a nearly decomposable system $T_{b i j}$ is small compared to $T_{w i}+T_{w j}$, for all $i$ and $j$. The calculation of these transmissions thus allows verification of a proposed grouping of variables into subsystems. The last question remains - how does infer a proposed grouping from the observations? A reasonable measure of the effect of $x_{i}$ on $x_{j}$ one time increment later is the normalized transmission $t_{i j}$

$$
t_{i j}=\frac{T\left(x_{i}: x_{j}^{\prime}\right)}{H\left(x_{j}^{\prime}\right)}
$$

According to above mentioned equations it is necessary to calculate the entropy of all state variables and joint entropy of pairs $\left(x_{i}, x_{j}^{\prime}\right) \quad$ where $i=1,2,3,4$ and $j=1,2,3,4$. To calculate normalized transmissions $t_{i j}$ between states of complex systems equation (11) is used and all these values are written in the table (see Table $6)$.

\begin{tabular}{|c|c|c|c|c|c|}
\hline & \multirow{2}{*}{$i$} & \multicolumn{4}{|c|}{$x_{j}^{\prime}$} \\
\hline & & $x_{1}^{\prime}$ & $x_{2}^{\prime}$ & $x_{3}^{\prime}$ & $x_{4}^{\prime}$ \\
\hline \multirow{4}{*}{$x_{i}$} & $x_{1}$ & 0,7955 & 0,5328 & 0,2573 & 0,1328 \\
\hline & $x_{2}$ & 0,5410 & 0,7416 & 0,1415 & 0,2416 \\
\hline & $x_{3}$ & 0,2629 & 0,1427 & 0,7832 & 0,5427 \\
\hline & $x_{4}$ & 0,1410 & 0,2416 & 0,5415 & 0,7416 \\
\hline
\end{tabular}

Table 6 Normalized transmission $t_{i j}$ between states of system

According to table (see Table 6), which contains calculated values of $t_{i j}$ we are able to decide whether complex system is decomposable intoto subsystems. According to values from table (see Table 6), it is clear that a complex system is nearly decomposable into subsystems $S^{1} \quad$ and $S^{2}, \quad$ where $S^{1}=\left\{x_{1}, x_{2}\right\}$ and $S^{2}=\left\{x_{3}, x_{4}\right\}$. This is only the initial idea about complex system and his subsystems, which however must be tested. Based on [8], the complex system is decomposable into subsystems, if couplings between variables that make up the subsystem are stronger than the couplings between separate subsystems. In our case, this fact can be verified only by calculate the transmissions using equations (8) and (9). Then for subsystem $S^{1}$ is $T_{w_{1}}=T\left(x_{1}: x_{1}^{\prime}: x_{2}: x_{2}^{\prime}\right)=13,47 \mathrm{bit} /$ sample time and for $S^{2}$ is $T_{w_{2}}=T\left(x_{3}: x_{4}^{\prime}: x_{3}: x_{4}^{\prime}\right)=13,63 \mathrm{bit} /$ sample time. $T_{w_{1}}$ and $T_{w_{2}}$ are transmissions that measures the nonindependance of all variables in the subsystem $S^{1}$ and $S^{2}$ over one time increment - sample time. It remains only to calculate the strength of coupling between subsystems $S^{1}$ and $S^{2}$ using equation (9). Then $T_{b_{12}}=T\left(\left(S_{1}, S_{1}^{\prime}\right):\left(S_{2}, S_{2}^{\prime}\right)\right)=\left(\left(x_{1}^{\prime}, x_{1}, x_{2}, x_{2}^{\prime}\right):\left(x_{3}, x_{4}^{\prime}, x_{3}, x_{4}^{\prime}\right)\right)$ $T_{b_{12}}=5,47 \mathrm{bit} /$ sample time . Since $T_{b_{12}} \ll T_{w_{1}}+T_{w_{2}} \Rightarrow$ $5,47 \ll 13,47+16,63$, the calculations confirm that the complex system is indeed nearly decomposable into subsystems $S^{1}$ and $S^{2}$.

\subsection{Decentralized control of complex system}

After decomposition of complex system into subsystems, we are able to design decentralized controller for each subsystem. In this section, two basic methods of decentralized control are introduced, namely decentralized control with quadratic criterion (partially decentralized control) and totally decentralized control.

\subsubsection{Decentralized control with quadratic criterion}

If we have complex system (5), so we know that is decomposable into two subsystems and thus global criterion is $J=\sum_{i=1}^{2} J_{i}$, while the local criterion is $J_{i}=\frac{1}{2} \int_{0}^{\infty}\left(\left\langle x_{i}(t), Q_{i} x_{i}(t)\right\rangle+\left\langle u_{i}(t), R_{i} u_{i}(t)\right\rangle\right) d t$, for $i=1,2$. Control strategy for first subsystem is in the form $u_{1}(t)=-R_{1}^{-1} B_{1}^{T} K_{1} \bar{x}_{1}(t)+R_{1}^{-1} B_{1}^{T} h_{1}$ and for second subsystem in the form $u_{2}(t)=-R_{2}^{-1} B_{2}^{T} K_{2} \bar{x}_{2}(t)+R_{2}^{-1} B_{2}^{T} h_{2}$, where $K_{1}$ and $K_{2}$ are solution of Riccati equations (12)

$$
\dot{K}_{i}=-K_{i} A_{i}-A_{i}^{T} K_{i}+K_{i} B_{i} R_{i}^{-1} B_{i}^{T} K_{i}-Q_{i}
$$

which are calculated off-line, $h_{1}$ and $h_{2}$ are solution of (13) and are calculated on-line.

$$
\begin{aligned}
\dot{h}_{i} & =-\left(A_{i}-B_{i} R_{i}^{-1} B_{i}^{T} K_{i}\right)^{T} h_{i}+ \\
& +K_{i} \sum_{\substack{j=1 \\
j \neq i}}^{N} A_{i j} x_{j}(t)+\sum_{\substack{j=1 \\
j \neq i}}^{N} A_{j i}^{T}\left(K_{j} x_{j}(t)-h_{j}\right)
\end{aligned}
$$

Proposed decentralized control with quadratic criterion can then be applied to control complex system (5). The main task of this control strategy is to convert the system from given initial conditions to steady state with minimizing a given criterion function. It should be noted, that control is designed to compensate the effect of interactions that exist between subsystems, like compensation of measurable disturbances in optimal control systems. States of complex system controlled by decentralized control with quadratic criterion are shown on figure (see Fig. 4). 


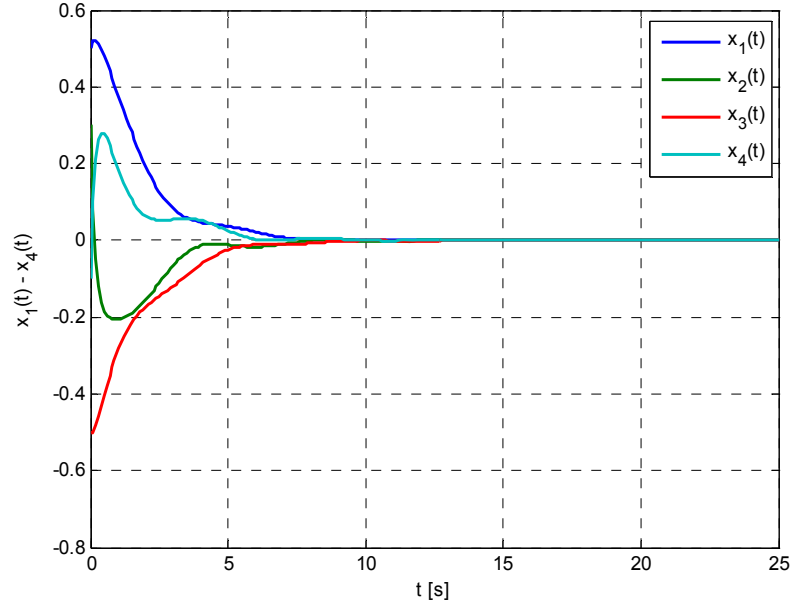

Fig. 4 States of complex system - decentralized control with quadratic criterion

\subsubsection{Totally decentralized control}

If we have complex system (5), so we know that is decomposable into two subsystems and thus global criterion is $J=\sum_{i=1}^{2} J_{i}$, while the local criterion is $J_{i}=\frac{1}{2} \int_{0}^{\infty}\left(\left\langle x_{i}(t), Q_{i} x_{i}(t)\right\rangle+\left\langle u_{i}(t), R_{i} u_{i}(t)\right\rangle\right) d t$, for $i=1,2$. Control strategy for first subsystem is in the form $u_{1}(t)=-R_{1}^{-1} B_{1}^{T} K_{1} \bar{x}_{1}(t)$ and for second subsystem in the form $u_{2}(t)=-R_{2}^{-1} B_{2}^{T} K_{2} \bar{x}_{2}(t)$, where $K_{1}$ and $K_{2}$ are solution of Riccati equations (12).

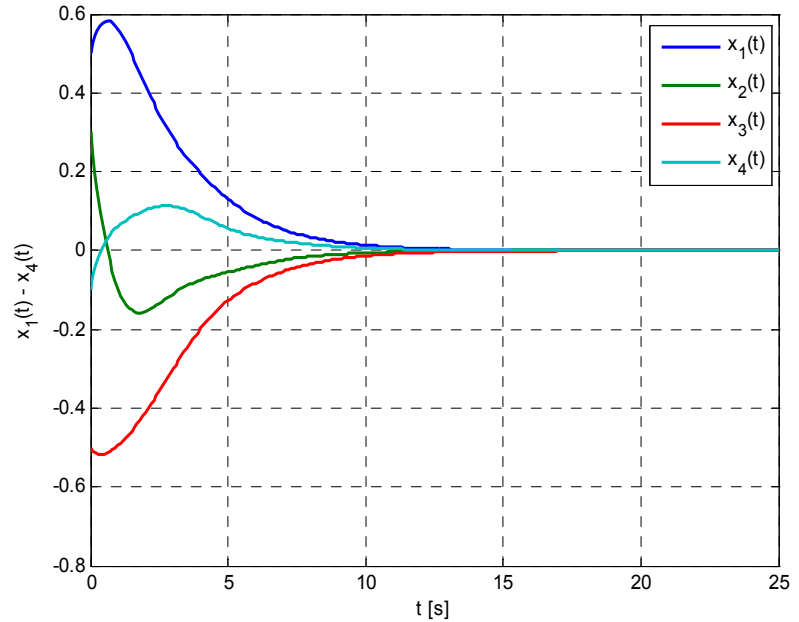

Fig. 5 States of complex system - totally decentralized control

States of complex system, which is controlled by totally decentralized control, are shown on figure (see Fig. 5). In this method of decentralized control, subsystems are considered as completely independence i.e. without interactions, while in a complex system are present. The disadvantage of this method is that it must be ensured not only the stability of subsystems, but also the stability of a complex system as a whole.

\subsection{Decentralized control and information theory}

As seen on figure (see Fig.4 and Fig. 5) from previous chapter, both methods of decentralized control convert complex system from specified initial conditions to steady state with minimizing a given criterion function. Let's look on these methods from information theory point of view. For better review and subsequent analysis will be better, that we look on internal structure of complex system (5), as shown on figure (see Fig. 6) [9].

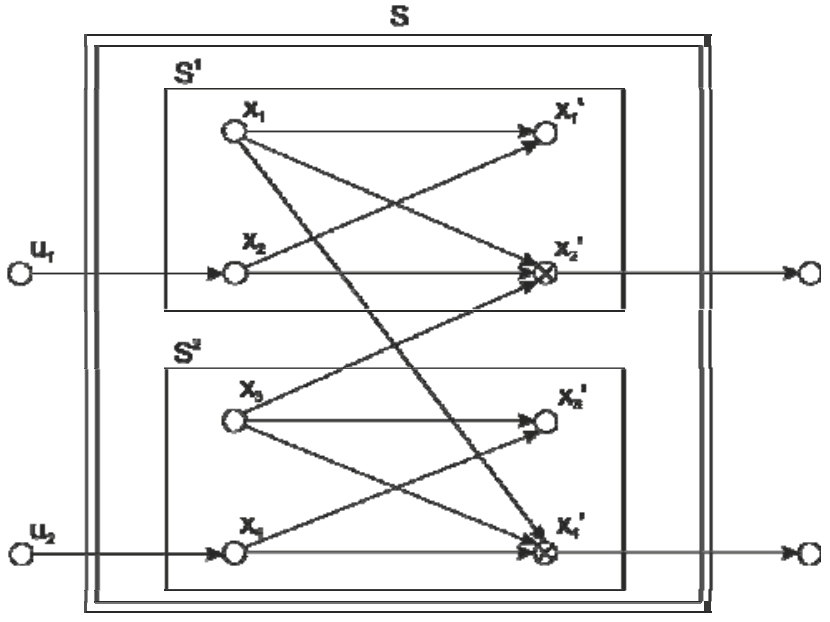

Fig. 6 Internal structure of complex system - information theory point of view

Figure (see Fig. 6) captures the internal structure of a complex system in discrete form (6) if the system is deterministic and its evolution can be represent by deterministic function $x(k+1)=f(x(k))$.

In this case Deterministic Partition Law of Information Rates (DPLIR) proposed by Roger C. Conant [2] is an appropriate tool to analyze complex systems in terms of information and information structure. Our complex system (5) respectively (6) is dynamic system, which changing its properties in time, so we will try to adapt this law to its properties [9]. Of course we assume that the complex system is decomposed into subsystems as shown on the figure (see Fig. 6) [9].

Let's start with the first subsystem, labelled as $S^{1}$. According to the figure (see Fig. 6), system contains internal and output variables. Sets of output variables are denoted as $S_{0}^{i}$ and sets of internal variables are denoted as $S_{\mathrm{int}}^{i}$ for $i$-th subsystem. So $S_{0}^{1}=\left\{x_{2}^{\prime}\right\}, S_{\mathrm{int}}^{1}=\left\{x_{1}, x_{2}, x_{1}^{\prime}\right\}$ and $S^{1}=\left\{x_{1}, x_{2}, x_{1}^{\prime}, x_{2}^{\prime}\right\}$ for the first subsystem $S^{1}$. For total rate (of information flow) $F^{1}$ in the first subsystem we can write

$F^{1}=\sum_{j} H\left(x_{j}^{1}\right)=H\left(x_{1}\right)+H\left(x_{2}\right)+H\left(x_{1}^{\prime}\right)+H\left(x_{2}^{\prime}\right)$

Throughput rate $F_{t}^{1}$ can be expressed as

$F_{t}^{1}=H\left(S_{0}^{1}\right)=H\left(x_{2}^{\prime}\right)$ 
For blockage rate $F_{b}^{1}$ we obtain

$$
\begin{aligned}
F_{b}^{1} & =H\left(S_{\mathrm{int}}^{1} \mid S_{0}^{1}\right)=H\left(S_{\mathrm{int}}^{1}, S_{0}^{1}\right)-H\left(S_{0}^{1}\right)= \\
& =H\left(x_{1}, x_{2}, x_{1}^{\prime}, x_{2}^{\prime}\right)-H\left(x_{2}^{\prime}\right)
\end{aligned}
$$

Finally, coordination rate $F_{c}^{1}$ (of information flow) in the first subsystem can be written as

$$
\begin{aligned}
F_{c}^{1} & =H\left(x_{1}\right)+H\left(x_{2}\right)+H\left(x_{1}^{\prime}\right)+H\left(x_{2}^{\prime}\right)-\left[H\left(x_{1}\right)+\right. \\
& \left.+H\left(x_{2}\right)+H\left(x_{1}^{\prime}\right)+H\left(x_{2}^{\prime}\right)-T\left(x_{1}: x_{2}: x_{1}^{\prime}: x_{2}^{\prime}\right)\right]= \\
& =T\left(x_{1}: x_{2}: x_{1}^{\prime}: x_{2}^{\prime}\right)
\end{aligned}
$$

Second subsystem denoted as $S^{2}$ have output and internal variables, that forming a sets $S_{0}^{2}=\left\{x_{4}^{\prime}\right\}$ and $S_{\text {int }}^{2}=\left\{x_{3}, x_{4}, x_{3}^{\prime}\right\}$. For total rate (of information flow) $F^{2}$ in the first subsystem we can write

$$
F^{2}=\sum_{j} H\left(x_{j}^{2}\right)=H\left(x_{3}\right)+H\left(x_{4}\right)+H\left(x_{3}^{\prime}\right)+H\left(x_{4}^{\prime}\right)
$$

Throughput rate $F_{t}^{2}$ can be expressed as

$$
F_{t}^{2}=H\left(S_{0}^{2}\right)=H\left(x_{4}^{\prime}\right)
$$

For blockage rate $F_{b}^{2}$ we obtain

$$
\begin{aligned}
F_{b}^{2} & =H\left(S_{\mathrm{int}}^{2} \mid S_{0}^{2}\right)=H\left(S_{\mathrm{int}}^{2}, S_{0}^{2}\right)-H\left(S_{0}^{2}\right) \\
& =H\left(x_{3}, x_{4}, x_{3}^{\prime}, x_{4}^{\prime}\right)-H\left(x_{4}^{\prime}\right)
\end{aligned}
$$

Finally, coordination rate $F_{c}^{2}$ (of information flow) in the first subsystem can be written as

$$
\begin{aligned}
F_{c}^{2} & =H\left(x_{3}\right)+H\left(x_{4}\right)+H\left(x_{3}^{\prime}\right)+H\left(x_{4}^{\prime}\right)-\left[H\left(x_{3}\right)+\right. \\
& \left.+H\left(x_{4}\right)+H\left(x_{3}^{\prime}\right)+H\left(x_{4}^{\prime}\right)-T\left(x_{3}: x_{4}: x_{3}^{\prime}: x_{4}^{\prime}\right)\right]= \\
& =T\left(x_{3}: x_{4}: x_{3}^{\prime}: x_{4}^{\prime}\right)
\end{aligned}
$$

Table (see Table 7) shows and summarizes results of computation of Deterministic Partition Law of Information Rates for Dynamical Systems (DPLIRDS) derived in [9]. Units for all rates are bit per sample time.

Table 7 Decentralized control in the terms of Deterministic Partition Law of Information Rates

\begin{tabular}{||c||c|c|c|c||}
\hline \hline $\begin{array}{c}\text { Decentralized } \\
\text { control }\end{array}$ & $F^{1}$ & $F_{t}^{1}$ & $F_{b}^{1}$ & $F_{c}^{1}$ \\
\hline Partially & 7,9770 & 1,9063 & 0,6382 & 5,5325 \\
\hline Totally & 11,681 & 2,7987 & 0,9549 & 7,9276 \\
\hline & $F^{2}$ & $F_{t}^{2}$ & $F_{b}^{2}$ & $F_{c}^{2}$ \\
\hline \hline Partially & 7,6910 & 1,6625 & 0,8075 & 5,2210 \\
\hline \hline Totally & 12,435 & 3,0909 & 0,8195 & 8,5250 \\
\hline \hline
\end{tabular}

\section{CONCLUSIONS}

Based on above derived and obtained law we can draw some remarks and conclusions. Consider figure (see Fig. 6 ), if we have full knowledge of inputs, then each state can be seen as output of communication channel. Uncertainty of output of communication channel can be affected only by change of the statistical properties of its input, which in our case corresponds to two different methods of decentralized control. We considered that uncertainty might be represented by computation of entropy. Figures (see Fig. 4 and Fig. 5) shown that uncertainties of all states with partially decentralized control are smaller then with totally decentralized control. The sum of all these uncertainties corresponds with total rate (of information flow). Of course not every state of the system must be its output and that is why the throughput rate only measures uncertainty of outputs variables. Blockage rate in control systems is negligible, because control systems don't process information directly and blocking of irrelevant information in the internal structure of system do not play major role, as confirmed by our results (see Table 7). Coordination rate has the greatest value of all rates. The main reason is in the decomposition, because the main idea of decomposition as mentioned above, assumes that the relationship between variables within the subsystem must be much larger than relationship between whole subsystems. The obtained results suggest that in partially decentralized control coordination rate is much smaller than in totally decentralized control. The reason is very simple. In partially decentralized control exists information link between controllers, i.e. controller for first subsystem has information from controller of second subsystem and vice versa. On the other hand in totally decentralized control this link no exists, so decentralized controllers control subsystems as independent parts and that is why the coordination rate must increase. Similar conclusions can be done from comparison of numerical criteria of control performance quality. But these criteria cannot explain why these differences occur and method presented in this paper can explain this.

\section{ACKNOWLEDGMENTS}

This publication is the result of the project implementation Centre of Information and Communication Technologies for Knowledge System (project number: 26220120020) supported by the Research \& Development Operational Programme funded by the ERDF.

\section{REFERENCES}

[1] ASHBY, W. R.: Requisite variety and its implications for the control of complex systems. Cybernetica, vol. 1, no. 1, pp. 83-99.

[2] CONANT, R. C.: Laws of information which govern systems. In: IEEE Transactions on systems science and cybernetics, vol. smc-6, no. 4, 1976, pp. 240 255.

[3] ASHBY, W. R.: Kybernetika. Praha: Orbis, 1961, $372 \mathrm{p}$. 
[4] KICKERT, W. J. M. - BERTRAND, J-W. M. PRAAGMAN, J.: Some Comments on Cybernetics and Control. In: IEEE Transactions on systems, man, and cybernetics, vol. smc-8, no. 11, November 1978, pp. 805-807.

[5] VENTCEL'OVÁ, J. S.: Theory of probability. Bratislava: Alfa, 1973, 524 p.

[6] ASHBY, W. R.: Measuring the internal information exchange in a system. In: Cybernetica, vol. 8, 1965, pp. 5-22.

[7] SARNOVSKÝ, J.: Control of complex system, 2. revised issue, Bratislava: Alfa, 1988, 340 p.

[8] CONANT, R. C.: Detecting subsystems of a complex system. In: IEEE Transactions on systems, man and cybernetics, vol. smc-2, no. 4, 1972, pp. 550-553.

[9] POPOVIČ, L'.: Principle of requisite variety in control systems. Dissertation thesis. Košice: The Technical University of Košice, Faculty of Electrical Engineering and Informatics, 2010, 137 p.

Received November 2, 2010, accepted February 21, 2011

\section{BIOGRAPHIES}

Luboš Popovič was born on 5.6.1984. In 2007 he graduated (MSc) at the Department of Cybernetics and
Artificial Intelligence of the Faculty of Electrical Engineering and Informatics at Technical University in Košice. Since 2007 he is a PhD student at the Department of Cybernetics and Artificial Intelligence. His PhD thesis in the field of Cybernetics; is "Principle of requisite variety in control systems". His scientific research is focusing on dynamic and control of complex and hybrid systems.

Ján Sarnovský was born on 23.3.1945. He received his MSc. in the field of Automation at Electrotechnical faculty of Slovak Technical University in Bratislava in 1968. In. 1980 he defended his doctoral candidate thesis and obtained scientific degree CSc. He obtained Professor of Technical Cybernetics in 1993. From 1989 he is a head of Department of Cybernetics and Artificial Intelligence of the Faculty of Electrical Engineering and Informatics at Technical University in Košice. He is coordinator of some national scientific grants. His scientific research is focusing on control of dynamic systems and multi-agent systems.

Vratislav Hladký was born on 12.5.1968. In 1991 he graduated (MSc) at the Department of Cybernetics and Artificial Intelligence of the Faculty of Electrical Engineering and Informatics at Technical University in Košice. He defended his $\mathrm{PhD}$ in the field of Control engineering and automation in 1996. His scientific research is focusing on dynamic and control of hybrid systems. 